La Revue

des Droits

de l'Homme

\section{La Revue des droits de l'homme}

Revue du Centre de recherches et d'études sur les droits fondamentaux

12 | 2017

Revue des droits de l'homme - $\mathrm{N}^{\circ} 12$

Les « missions égalité entre les hommes et les femmes » dans les universités : quelles évolutions depuis la loi relative à l'enseignement supérieur et à la recherche du 22 juillet 2013 ?

\title{
Béatrice Delzangles
}

\section{(2) OpenEdition}

Journals

Édition électronique

URL : http://journals.openedition.org/revdh/3258

DOI : $10.4000 /$ revdh.3258

ISSN : 2264-119X

Éditeur

Centre de recherches et d'études sur les droits fondamentaux

Référence électronique

Béatrice Delzangles, «Les «missions égalité entre les hommes et les femmes » dans les universités : quelles évolutions depuis la loi relative à l'enseignement supérieur et à la recherche du 22 juillet 2013 ? », La Revue des droits de l'homme [En ligne], 12 | 2017, mis en ligne le 13 juillet 2017, consulté le 08 juillet 2020. URL : http://journals.openedition.org/revdh/3258; DOI : https://doi.org/10.4000/revdh. 3258

Ce document a été généré automatiquement le 8 juillet 2020

Tous droits réservés 


\title{
Les « missions égalité entre les hommes et les femmes » dans les universités : quelles évolutions depuis la loi relative à l'enseignement supérieur et à la recherche du 22 juillet 2013 ?
}

\author{
Béatrice Delzangles
}

1 Comment l'université peut-elle penser la cause des femmes ? $^{1}$ Plusieurs démarches existent : renforcer leur présence dans les instances de gouvernance des établissements d'enseignement et des organismes de recherche, favoriser la parité dans tous les organes décisionnels, rendre plus égalitaire le déroulement des carrières, augmenter la place des femmes dans les filières scientifiques ou encore lutter résolument contre le harcèlement sexuel. Une partie de ces mesures ont été prévues par la loi relative à l'enseignement supérieur et à la recherche du 22 juillet 2013, dite loi Fioraso. Certaines ont été renforcées par la loi égalité et citoyenneté du 27 janvier $2017^{2}$. Parmi ces mesures, il en une plus transversale qui oblige désormais chaque université à mettre en place une structure dédiée à l'égalité femmes-hommes. L'article L. 712-2 alinéa $10 \mathrm{du}$ code de l'éducation, tel que modifié par la loi Fioraso, dispose en effet que le président de l'université « installe, sur proposition conjointe du conseil d'administration et du conseil académique, une mission 'égalité entre les hommes et les femmes' ». Si cette disposition concerne uniquement la gouvernance des universités, plusieurs décrets adoptés ultérieurement étendent cette exigence à d'autres établissements publics à caractère scientifique, culturel et professionnel ayant un statut particulier ${ }^{3}$. Partant, l'obligation de mettre en place des missions égalité femmes-hommes ne s'adresse plus exclusivement aux universités mais plus largement aux établissements publics d'enseignement supérieur (EPES) bien que tous ne soient pas (encore ?) concernés ${ }^{4}$. 
2 L'obligation pour chaque université et établissement à caractère scientifique, culturel et professionnel concernés d'identifier une structure ou une personne dédiée à l'égalité femmes-hommes témoigne de la prise de conscience par les pouvoirs publics de la persistance des inégalités entre les sexes dans le monde académique et de la volonté du législateur d'agir. Elle est le résultat d'un long processus mené en amont et impliquant des associations clés du monde de l'enseignement supérieur et de la recherche telles que la Conférence des Grandes Écoles (CGE), la Conférence des Présidents d'Université (CPU), la Conférence des directeurs des écoles françaises d'ingénieurs (Cdefi) ${ }^{5}$ ou encore la Conférence Permanente des chargé.e.s de mission Égalité et Diversité (CPED) ${ }^{6}$ ou l'Association Nationale des Études Féministes (ANEF). Pourtant, en 2013, lors de l'élaboration de la loi relative à l'enseignement supérieur et à la recherche, le besoin de défendre la cause des femmes dans le monde académique était loin d'être une évidence pour tous et toutes. Des oppositions se sont en effet manifestées à l'occasion de l'examen des amendements proposés au nom de la Délégation aux droits des femmes et à l'égalité des chances entre les hommes et les femmes de l'Assemblée nationale, une députée notamment craignant l'« excès de parité et d'égalité " ${ }^{7}$.

3 Quatre ans après l'adoption de la loi Fioraso et la modification du code de l'éducation qui en a résulté, il reste difficile et peut-être prématuré de se prononcer sur l'efficacité des missions égalité femmes-hommes installées dans les différents établissements du supérieur. L'application concrète et effective de l'article L 712-2 alinéa 10 par les établissements concernés ainsi que la manière dont ces missions s'exercent en pratique sont des questions relevant davantage d'une étude sociologique. Cependant, la présente analyse juridique des missions égalité femmes-hommes se propose d'apporter des éléments d'explication susceptibles d'éclairer les raisons de la réussite ou, le cas échéant, de l'échec de ce dispositif en revenant, notamment, sur la genèse des missions égalité femmes-hommes à l'université, leurs fondements juridiques, l'évolution de leur nature et de leurs fonctions depuis les années 2000, avant qu'elles ne deviennent obligatoires, et en identifiant leurs forces et leurs faiblesses.

4 Les missions égalité femmes-hommes créées dans les établissements d'enseignement supérieur consistent à confier à des structures et/ou à des personnes désignées la défense de la cause des femmes au sein de l'établissement afin que celle-ci ne relève plus seulement d'initiatives ou de revendications individuelles. Ces missions sont censées contribuer à la formalisation d'une stratégie et d'un plan d'action en matière d'égalité des sexes propres à chaque établissement et à pérenniser la promotion de l'égalité femmes-hommes dans celui-ci. Partant, à l'instar de l'État, les établissements d'enseignement supérieur et de recherche pensent la cause des femmes en institutionnalisant celle-ci ${ }^{8}$. Demeurée un temps à la seule appréciation des établissements (I), la présence d'une mission égalité femmes-hommes est désormais obligatoire pour toutes les universités et un nombre croissant d'établissements d'enseignement supérieur (II). Cette institutionnalisation progressive favorise-t-elle la lutte contre les inégalités entre les sexes ou s'agit-il d'une amélioration en trompe l'œil ? 


\section{I - Du choix des établissements d'enseignement supérieur de nommer un.e chargé.e de mission égalité femmes-hommes...}

5 Les chargé.e.s de mission égalité femmes-hommes sont apparu.e.s au début des années 2000 au sein de certains établissements d'enseignement supérieur et de recherche soucieux de l'égalité des sexes dans leur organisation (A). Par la suite, cette fonction a progressivement été placée au centre des dispositifs initiés ultérieurement par les acteurs du monde de l'enseignement supérieur et de la recherche (B).

\section{A - L'apparition des chargé.e.s de mission égalité femmes-hommes}

6 En 2000, une convention interministérielle pour la promotion de l'égalité des chances entre les filles et les garçons, les femmes et les hommes dans le système éducatif ${ }^{9}$ a été adoptée afin d'encourager l'élaboration et la mise en œuvre d'une politique globale d'égalité des chances entre les sexes en direction de tous les acteurs, du préélémentaire à l'enseignement supérieur, de la formation initiale à la formation tout au long de la vie. En tant que maillon du système éducatif, les établissements d'enseignement supérieur ont été associés à ce processus. La convention prévoyait notamment qu'une politique d'égalité des chances entre les femmes et les hommes soit intégrée dans « les projets d'établissements d'enseignement (...) supérieur » ainsi que dans "les contrats quadriennaux des établissements universitaires » afin de "renforcer les outils de promotion de l'égalité et la formation des acteurs ». Rien n'était dit à l'époque sur la mise en place, au sein des EPES, d'organes, de services, de personnes ou de missions dédiés. Cependant, les nombreuses mesures visant l'enseignement supérieur identifiées par les ministres signataires de cette convention soulevaient déjà la question des moyens humains mobilisés dans chaque établissement afin d'en assurer la mise en œuvre concrète. Lorsque cette convention interministérielle fut reconduite en 2006, ce fut l'occasion d'y faire apparaître l'engagement de «nommer au sein des établissements d'enseignement, des correspondant(e)s égalité en charge de la coordination des actions en matière d'égalité entre les sexes dans leur établissement respectif $»^{10}$.

7 Dans les faits, certains établissements avaient déjà nommé des chargé.e.s de mission égalité femmes-hommes entre 2000 et $2006^{11}$ grâce, notamment, à des financements obtenus du Fonds social européen (FSE). Ce Fonds a constitué un autre dispositif déterminant dans l'apparition des premier.e.s chargé.e.s de mission égalité femmeshommes dans les EPES. Les conventions interministérielles précitées y faisaient d'ailleurs expressément référence ${ }^{12}$. Le règlement relatif au FSE pour la période 2000-2006 ${ }^{13}$ insistait sur la nécessité d'intégrer la promotion de l'égalité des sexes dans toutes les actions soutenues par le FSE là où le règlement précédent (pour la période 1994-1999) se focalisait sur l'égalité des chances pour les femmes et les hommes sur le marché du travail ${ }^{14}$. À partir des années 2000, le FSE a donc développé une approche transversale de l'égalité des sexes de sorte que toute action en lien avec cet objectif pouvait prétendre être soutenue par ce fonds structurel ${ }^{15}$.

Le financement des premier.e.s chargé.e.s de mission égalité femmes-hommes dans les EPES ont pu s'inscrire sans difficulté dans cette nouvelle approche européenne. Elles et 
ils soutenaient l'accès des femmes au marché du travail, notamment en promouvant la mixité des filières ou en luttant contre les freins à la carrière des enseignanteschercheures. Plus largement, elles et ils témoignaient de l'inclusion de l'égalité femmes-hommes dans toutes les politiques publiques nationales, y compris celles relatives à l'enseignement supérieur et à la recherche et s'inscrivaient ainsi dans l'approche intégrée de l'égalité des genres défendue par l'Union européenne. L'apparition des chargé.e.s de mission concomitamment aux versements du FSE puis la disparition de certain.e.s avec la fin des versements témoignent de la part non négligeable prise par la Communauté européenne à l'époque, et plus spécifiquement par les fonds structurels, dans la promotion et la mise en œuvre de l'égalité femmeshommes dans les établissements d'enseignement supérieur.

\section{B - Le rôle croissant accordé aux chargé.e.s de mission égalité femmes-hommes}

9 À partir de 2005, les nominations de chargé.e.s de mission se sont plutôt inscrites dans une réflexion dédiée spécifiquement à l'enseignement supérieur et à la recherche. La Conférence des Grandes Écoles (CGE) signa cette année-là la Convention pour la promotion de l'égalité entre les hommes et les femmes ${ }^{16}$ avec la ministre de la parité et de l'égalité professionnelle, Nicole Ameline, dans laquelle était prévue la désignation d' " un référent 'égalité hommes/femmes' au sein des Écoles ». Quatre ans plus tard, la Conférence des Présidents d'Université (CPU) adopta la première Charte pour l'égalité entre Femmes et Hommes dans les établissements d'enseignement supérieur et de recherche ${ }^{17}$ qui préconisait qu' « une Mission pour l'Égalité entre les Femmes et les Hommes avec à sa tête une chargée de mission ${ }^{18}$ soit "responsable de la mise en œuvre » de la politique de l'université signataire en matière d'égalité entre les sexes. Ce texte faisait ainsi de la mission égalité femmes-hommes le relais institutionnel indispensable à l'application du dispositif prévu par l'université en la matière. Plusieurs universités ont alors désigné une mission dédiée à l'égalité femmes-hommes ou ont intégré cette mission dans une fonction plus large créée à cette occasion ou déjà existante. À l'époque, «très peu de chargé.e.s de mission alors nommé.e.s sont des professionnel.le.s de la question de genre, une particularité de cette deuxième vague. Ils et elles sont mathématicien.ne.s, biologistes, juristes ou physicien.ne.s $»^{19}$.

D'essence synallagmatique ou concertée, les conventions interministérielles, la convention CGE et la Charte CPU sur l'égalité femmes-hommes contiennent principalement des dispositions déclaratoires ou programmatiques, de simples directives destinées à guider l'action de leurs signataires sans être prescriptives. Elles s'apparentent, en cela, à des actes non-juridiques ${ }^{20}$ relevant de la soft law ${ }^{21}$. Bien que non contraignants, ces textes ont permis de formaliser l'engagement des deux Conférences en faveur de l'égalité femmes-hommes et de donner une feuille de route aux différents EPES dans ce domaine. La démarche restait cependant hétérogène au sein du monde de l'enseignement supérieur et de la recherche avec deux textes distincts qui, d'ailleurs, ne reposaient pas sur la même logique. La Convention de 2005 visait à favoriser une coopération entre le ministère de la parité et de l'égalité professionnelle et la CGE ainsi qu'à mettre en place des dispositifs partagés entre ces deux organisations signataires en vue de "sensibiliser à la question de l'égalité face aux études et à la vie professionnelle", de "veiller au respect de l'égalité entre étudiantes et étudiants dans les écoles membres de la Conférence » et de " participer au 
développement de l'égalité professionnelle entre femmes et hommes tant dans le secteur privé que dans le secteur public ». Elle faisait ainsi de la CGE l'institution de premier plan censée intervenir auprès des grandes écoles membres pour développer «une politique d'incitation destinée à favoriser l'Égalité ». Différente, la première Charte pour l'égalité entre les femmes et les hommes de la CPU s'adressait directement aux universités membres de la CPU qui, après avoir spontanément accepté de signer ce texte, s'engageaient à respecter les recommandations énoncées.

11 Finalement, c'est cette dernière approche qui fut privilégiée en 2013, lors de l'adoption d'une nouvelle Charte pour l'égalité entre Femmes et Hommes dans les établissements d'enseignement supérieur et de recherche associant le ministère de l'enseignement supérieur et de la recherche, le ministère du droit des femmes, la CPU mais également la CGE et la Conférence des directeurs des écoles françaises d'ingénieurs (Cdefi). Parmi les engagements communs aux établissements acceptant de signer cette charte universités, grandes écoles ou écoles d'ingénieur -, il y a celui de nommer « un.e. référent.e " égalité femmes-hommes ${ }^{22}$. Ce texte commun est accompagné de deux chartes distinctes - l'une pour les universités et les écoles d'ingénieurs, l'autre pour les grandes écoles - venant préciser les modalités d'application des engagements « que les établissements sont invités à s'approprier et à mettre en œuvre ». Dans les deux cas, la ou le référent.e est clairement identifié.e comme celle ou celui chargé.e d'assurer la réalisation concrète de la politique égalité femmes-hommes de l'établissement. Il lui est donc confié un rôle central dans le dispositif ${ }^{23}$.

Bien que fondée sur des actes non contraignants jusqu'en 2013, la démarche en faveur de l'égalité femmes-hommes dans l'enseignement supérieur et la recherche s'est enrichie au fil des années, tant du point de vue de l'approche privilégiée que du contenu. Le passage de l'intitulé « convention » à "charte » témoigne de la volonté du monde académique de s'engager en faveur de l'égalité femmes-hommes. Parallèlement, depuis les années 2000 et la première convention interministérielle, la démarche se veut à la fois plus spécifique - avec un dispositif dédié à l'enseignement supérieur et à la recherche - et plus complète - avec une charte qui s'appliquent à l'ensemble des établissements du supérieur et de la recherche et qui implique toutes les missions de ces établissements et toutes leurs composantes. Enfin, le contenu du dispositif s'est " juridicisé " grâce à des résolutions plus précises s'inscrivant dans la durée.

En rendant obligatoire les missions égalité femmes-hommes, la loi Fioraso consacre-telle cette évolution?

\section{II - ... à l'obligation « d'installer une mission 'égalité entre les hommes et les femmes' » dans toutes les universités}

14 La loi Fioraso ayant rendu obligatoire pour toutes les universités et certains

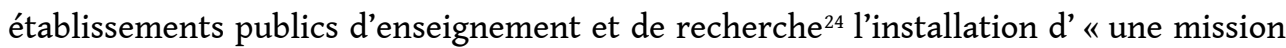
'égalité entre les hommes et les femmes'», celle-ci s'en trouve-t-elle mieux promue dans les établissements concernés ? Si la loi impose désormais la mise en place d'une structure dédiée, elle ne consacre pas la pratique qui s'était jusque-là développée, à savoir la désignation de chargé.e.s de mission ou de référent.e.s égalité femmeshommes (A). De même, en l'absence de précision du contenu de la mission égalité 
femmes-hommes et de ses objectifs, l'obligation qui pèse sur les EPES concernés est finalement peu contraignante (B).

\section{A. Référent.e ou mission : quel enjeu ?}

15 À l'origine, le projet de loi relative à l'enseignement supérieur et à la recherche ne prévoyait rien sur les missions égalité femmes-hommes. C'est lors du travail en commission des affaires culturelles et de l'éducation de l'Assemblée nationale, compétente pour étudier préalablement le texte proposé, qu'un amendement a été adopté afin de rendre obligatoire la nomination, par le président de l'université, d' "un.e chargé.e de mission 'Égalité entre les hommes et les femmes' »"25. A ce stade, déjà, cette initiative avait donné lieu à l'opposition de certains membres de la commission parlementaire qui voyait dans cette démarche coercitive une atteinte à l'autonomie des établissements d'enseignement supérieur et un manque de cohérence vis-à-vis des autres chargé.e.s de mission susceptibles d'être désigné.e.s par l'établissement sans avoir le même statut ${ }^{26}$.

Malgré des oppositions, le projet a maintenu dans un premier temps l'amendement proposé tout en le précisant. Le texte de la commission déposé le 16 mai 2013 prévoyait ainsi la nomination d'un.e chargé.e de mission «Égalité entre les femmes et les hommes" sur proposition conjointe du conseil d'administration et du conseil académique et dont la mission et les compétences seraient précisées par décret en Conseil d'Etat ${ }^{27}$. Cependant, lors du débat en séance publique à l'Assemblée nationale, et à l'initiative du gouvernement, la référence au chargé de mission et au décret a disparu pour être remplacée par une "mission égalité entre les hommes et les femmes». Il ressort des discussions parlementaires que le gouvernement de l'époque n'a pas souhaité imposer aux universités une charge de dépenses supplémentaires. Pour beaucoup, en effet, la désignation d'un.e chargé.e de mission serait revenue à créer un poste spécifique dans chaque établissement. Le législateur a donc préféré laisser aux universités le choix de redéployer une mission existante ou de créer une mission spécifique et/ou de la confier à un.e membre du personnel administratif ou à un.e enseignant.e-chercheur.e.

17 Cette réécriture du texte - version finalement retenue par le code de l'éducation - estelle moins ambitieuse que l'obligation initiale de désigner une personne référente? Une mission égalité femmes-hommes est susceptible de bénéficier de moyens humains voire d'un budget propre là où un.e chargé.e de mission unique ne dispose généralement que d'une décharge d'heures. C'est ainsi que certaines universités ont choisi de mettre en place un "pôle égalité ", c'est-à-dire un service central dédié à l'égalité des sexes ${ }^{28}$. Cependant, une mission n'est pas toujours aussi identifiable qu'un pôle, un.e vice président.e ou un.e chargé.e de mission égalité femmes-hommes. C'est le cas par exemple lorsqu'elle est rattachée à la Direction des ressources humaines (DRH). Or, la question de sa visibilité est essentielle, notamment lorsqu'il s'agit de recueillir le signalement de dysfonctionnements. En cas de harcèlement sexuel, par exemple, la victime doit pouvoir savoir à qui s'adresser et être assurée que la situation qu'elle dénonce entre bien dans le champ de compétences de la mission égalité femmeshommes ${ }^{29}$. De surcroit, le rattachement de celle-ci à certains services administratifs de l'établissement rend difficile un traitement de l'égalité femmes-hommes dans sa globalité. Ainsi, des problématiques essentielles telles que la lutte contre le 
harcèlement sexuel au sein de la communauté étudiante ou la prise en compte des études de genre dans les formations et/ou la recherche échappent totalement au service des ressources humaines.

Dans les faits, les missions égalité semblent peu à peu prendre de l'ampleur au sein des établissements d'enseignement supérieur. On constate en effet depuis la loi Fioraso une augmentation des référent.e.s, chargé.e.s de mission ou vice-président.e.s égalité au sein des EPES. Une majorité d'établissement d'enseignement supérieur ne disposant pas de mission égalité femmes-hommes au moment de l'entrée en vigueur de la loi semblent donc avoir choisi de s'y conformer en désignant une personne dédiée, s'inspirant ainsi de la pratique développée par plus de la moitié des EPES au moment de l'adoption de la loi. Selon la liste des membres de la Conférence Permanente des chargé.e.s de mission Egalité et Diversité (CPED) créée en 2001, soixante-deux universités (sur soixante-quinze) ont une mission égalité ayant pris attache avec ce réseau au $1^{\mathrm{er}}$ juin 2017, dont dix-neuf installées après la loi Fioraso ${ }^{30}$. Dans dix-neuf universités participant à cette Conférence, la mission Égalité femmes-hommes est portée par un.e vice-président.e dont neuf sont dédié.e.s à l'égalité et à la lutte contre les discriminations. Les autres vice-président.e.s ont un périmètre d'action élargi à la responsabilité sociale de l'université, aux ressources humaines, au développement durable, à la culture ou encore au patrimoine et aux finances. En revanche, les chargé.e.s de mission recensé.e.s ont une fonction dédiée à la lutte contre les discriminations et à la promotion de l'égalité et plus spécifiquement à l'égalité femmeshommes et/ou à la parité pour plus de la moitié d'entre elles/eux ${ }^{31}$.

Sans prétendre dresser un état des lieux exhaustif de la situation actuelle des missions égalité femmes-hommes, ces quelques chiffres attestent d'une augmentation de ces missions au sein des EPES ces dernières années. Cependant, si l'institutionnalisation de ces missions est aujourd'hui une réalité, la forme qu'elle peut prendre pose encore question. On constate en effet parmi les universités membres de la CPED une grande variété des statuts et des missions octroyées aux personnes en charge de l'égalité femmes-hommes, sans pouvoir dire s'il est plus efficace pour la cause des femmes d'être portée politiquement par un.e vice-président.e dont c'est une des fonctions parmi d'autres ou s'il est préférable qu'elle soit promue par un.e charg.é.e de mission spécifiquement dédié.e à la cause. En outre, l'efficacité de la mission égalité femmeshommes ne saurait dépendre uniquement du statut de celle ou celui qui l'exerce. La visibilité de la mission au sein de l'université, les moyens alloués, le soutien accordé de même que l'implication des personnes qui en ont la charge sont autant de facteurs qui en conditionnent la réussite. Là encore, ces facteurs varient considérablement d'un établissement à l'autre.

\section{B. L'absence de contenu précis donné à la mission égalité femmes- hommes}

20 Déjà du temps où les chargé.e.s de mission égalité femmes-hommes étaient librement désigné.e.s par les établissements d'enseignement supérieur qui le souhaitaient, la nature et le contenu de leurs fonctions étaient très variés. Le fait de rendre obligatoire les missions égalité femmes-hommes ne s'est pas accompagné d'une harmonisation de leur périmètre d'action ni de leurs objectifs. L'article L. 712-2 alinéa 10 du code de l'éducation ne dit rien, en effet, sur le rôle que doivent jouer ces missions une fois 
installées. De plus, la loi a renoncé à l'adoption d'un décret en Conseil d'État qui aurait pu en préciser le contenu et les compétences.

Soucieux de préserver l'autonomie des établissements d'enseignement supérieur et de recherche, le législateur a fait le choix de laisser à ces derniers une importante marge de manœuvre quant à la manière de promouvoir concrètement l'égalité entre les femmes et les hommes. S'il est vrai que l'absence de précision tenant aux actions à mener et aux dispositifs à mettre en place peut favoriser le dynamisme et l'innovation de certaines universités qui décident de prendre au sérieux la mission égalité femmeshommes, elle peut également permettre à d'autres de se contenter du strict minimum. Gage de souplesse, l'absence de contenu assigné à ces missions peut aussi en faire des coquilles vides.

Cette souplesse est également source de confusions quant à la délimitation des fonctions que doit exercer la mission égalité femmes-hommes. Dans le silence de la loi Fioraso, le plan d'action du ministère de l'éducation nationale, de l'enseignement supérieur et de la recherche, prenant lui-même appui sur la politique interministérielle pour l'égalité femmes-hommes formalisée en 2013, peut constituer la feuille de route des missions égalité femmes-hommes installées dans les universités ${ }^{32}$. Ce plan d'action s'est voulu ambitieux: pas moins de huit mesures ${ }^{33}$ déclinées en quarante actions concrètes ont été identifiées pour permettre la mise en place d'une politique globale d'égalité femmes-hommes dans les établissements d'enseignement supérieur prenant en compte les personnels, les étudiant.e.s et les savoirs scientifiques sur l'égalité et le genre $^{34}$. Cependant, ce plan d'action ministériel ne dit rien sur les missions égalité femmes-hommes des universités là où il aurait pu préciser le rôle de celles-ci dans sa mise en œuvre.

Il existe donc encore beaucoup d'incertitudes sur le contenu, le périmètre et les compétences des missions égalité femmes-hommes des universités. Certes, elles ont vu récemment leurs attributions précisées partiellement. La circulaire du 25 novembre 2015 relative à la prévention et aux traitements du harcèlement sexuel dans les établissements publics d'enseignement supérieur et de recherche leur confie en effet un rôle en la matière ${ }^{35}$. Elle précise par exemple que ces missions doivent inscrire la prévention du harcèlement sexuel dans les priorités du plan d'action de l'établissement en faveur de l'égalité des sexes et proposer des mesures d'accompagnement des victimes de harcèlement sexuel. Elle compte également la " mission égalité » parmi les instances susceptibles de signaler une situation de harcèlement sexuel auprès du responsable de l'établissement. Elle encourage par ailleurs les établissements d'enseignement supérieur «à solliciter l'expertise de (...) la mission égalité (...)» lorsqu'une procédure disciplinaire est engagée pour des faits de harcèlement sexuel ${ }^{36}$. La circulaire contribue ainsi à concrétiser le rôle de la mission égalité et/ou des référent.e.s égalité en leur confiant une fonction de proposition, d'alerte et d'expertise dans le dispositif de lutte contre le harcèlement sexuel à l'universités ${ }^{37}$.

Les missions égalité femmes-hommes installées dans les établissements d'enseignement supérieur restent variées. Ce constat est renforcé dans les établissements où l'égalité femmes-hommes est associée à d'autres fonctions telles que la lutte contre toutes les discriminations, la laïcité, la diversité, la responsabilité sociale de l'établissement, le développement durable, les ressources humaines, la culture ou encore le patrimoine et les finances. Le ministère lui-même tend à élargir leur périmètre d'action en renvoyant, dans des textes récents, à la «mission en faveur de l'égalité des sexes et de la lutte 
contre les discriminations » ou encore à " la personne référente en charge de l'égalité » ${ }^{38}$. Il est aussi désormais question d'inciter les établissements d'enseignement supérieur à candidater au « label diversité » mis en place en 2008 dans la fonction publique et au label «égalité professionnelle » effectif depuis 2016. Ici aussi, le critère du sexe a formellement disparu. La promotion de l'égalité femmes-hommes à l'université, y compris ses relais institutionnels que sont les missions égalité femmes-hommes, est concernée par le phénomène de "généralisation et d'objectivation des critères " de discriminations retenues pour définir des politiques publiques ${ }^{39}$. Elle témoigne, elle aussi, du glissement, voire de la dilution, de l'égalité des sexes (gender mainstreaming) dans l'égalité pour tous et la diversité (diversity mainstreaming) ${ }^{40}$.

Avec la loi Fioraso, les missions égalité femmes-hommes sont devenues obligatoires mais n'ont pas gagné en contenu. Cela dit, ce manque de précision peut s'avérer stratégique. Il a notamment permis d'éviter le débat sur le contenu et la manière d'exercer la mission égalité femmes-hommes, à la différence de l'institutionnalisation des études féministes qui, lui, a donné lieu à des tensions entre militantisme et recherche scientifique et entre action et théorie ${ }^{41}$. Ensuite, la mission égalité femmeshommes des universités et établissements assimilés doit se penser dans un ensemble normatif dépassant le cadre de la loi. Les missions étant désormais obligatoires, et face au vide laissé par le législateur quant à leur contenu, les personnes en charge de l'égalité femmes-hommes dans chaque établissement se tournent spontanément vers ce qui existe déjà en la matière : la Charte pour l'égalité entre Femmes et Hommes dans les établissements d'enseignement supérieur et de recherche, le plan d'action du ministère ou encore le réseau CPED. Par un effet mécanique, la loi imprécise favorise la mise en œuvre des normes de droit souple élaborées jusqu'ici en matière d'égalité femmeshommes dans l'enseignement supérieur et la recherche. Parallèlement, ces instruments de droit souple se mettent au service de l'effectivité de l'article L 712-2 alinéa 10. Une telle approche peut favoriser au sein de chaque établissement des actions concertées entre les différentes parties prenantes - étudiant.e.s, membre du personnel administratif et enseignant.e.s-chercheur.e, organisations partenaires telles que les associations, les administrations ou les entreprises. Elle peut aussi se montrer hasardeuse en entrainant une promotion de l'égalité femmes-hommes à géométrie variable au sein des différents établissements concernés.

\section{NOTES}

1. Pour paraphraser Anne RÉVILLARD qui pose cette question plus largement à propos de l'État, in La cause des femmes dans l'État. Une comparaison France-Québec, Presses universitaires de Grenoble, 2016, p. 16.

2. L'article 166 de la loi nº 2017-86 du 27 janvier 2017 prévoit que, pour tout jury de concours et comité de sélection de la fonction publique, la présidence soit confiée de manière alternée à un membre de chaque sexe (sauf dérogation prévue par décret en Conseil d'Etat).

3. Il en est ainsi pour le Conservatoire national des arts et métiers, l'Université Paris-Dauphine, l'Institut polytechnique de Bordeaux, l'École normale supérieure de Cachan, l'Université de 
Lorraine, l'Université de Nîmes (décret n 2014-1239 du 24 octobre 2014 relatif à des établissements publics à caractère scientifique, culturel et professionnel), CentraleSupélec (décret $n^{\circ}$ 2014-1679 du 30 décembre 2014, ), l'École nationale supérieure d'arts et métiers (décret $n^{\circ}$ 2015-40 du 20 janvier 2015), la communauté d'universités et établissements «Université fédérale de Toulouse Midi-Pyrénées » (décret $n^{\circ} 2015-663$ du 10 juin 2015) ou encore l'Institut d'études politiques de Paris (décret $n^{\circ} 2016-24$ du 18 janvier 2016).

4. Par exemple, le décret $n^{\circ} 2014-1239$ du 24 octobre 2014 relatif à des établissements publics à caractère scientifique, culturel et professionnel (op.cit.) n'étend pas cette obligation à l'Institut national d'histoire de l'art ni à l'École pratique des hautes études ou à l'École nationale supérieure maritime.

5. Chacune mène en son sein une démarche en faveur de l'égalité femmes-hommes. Ainsi, la CGE a créé en 2004 un groupe de travail égalité femmes-hommes au sein de sa commission «Diversité ». La CPU a procédé de même en 2011 en mettant en place un groupe de travail «Egalité Femmes/Hommes » au sein de sa commission « des moyens et des personnels ». La Cdefi compte, quant à elle, la promotion de l'égalité femmes-hommes parmi les travaux prioritaires de sa commission « Formation et Société ».

6. Il s'agit d'une association née en janvier 2011 afin de regrouper des chargé.e.s de mission et des vice-président.e.s en charge de l'égalité entre les femmes et les hommes dans leur établissement.

7. V. l'intervention de Mme Sophie Dion, députée Les Républicains, in Rapport du 16 mai 2013 de Vincent FELTESSE, Rapporteur du projet de loi relative à l'enseignement supérieur et à la recherche adoptée le 22 juillet 2013, http://www.assemblee-nationale.fr/14/rapports/r1042.asp, consulté le 5 juillet 2017.

8. Sur ce point, v. Anne RÉVILLARD, La cause des femmes dans l'Etat. Une comparaison France-Québec, op. cit.

9. Cette convention adoptée le 25 février 2000 s'adresse notamment «aux présidentes et présidents d'université ». Elle a été signée par la ministre de l'emploi et de la solidarité, le ministre de l'éducation nationale, de la recherche et de la technologie, le ministre de l'agriculture et de la pêche, la ministre déléguée chargée de l'enseignement scolaire, la secrétaire d'État aux droits des femmes et à la formation professionnelle (BO $\mathrm{n}^{\circ} 10$ de l'Education Nationale du 9 mars 2000, NOR: MENENE0000586X, http://www.education.gouv.fr/bo/2000/10/orga.htm, consultée le 5 juillet 2017). Le 8 mars 2002, les ministres de la justice, de l'enseignement professionnel, de la culture et de la communication, de l'équipement, des transports et du logement et de la recherche l'ont également signée.

10. Convention pour l'égalité entre les filles et les garçons, les femmes et les hommes, dans le système éducatif signée le 28 juin 2006 (BO n 5 du $1^{\text {er }}$ février 2007, NOR MENE0603248X, http:// www.education.gouv.fr/bo/2007/5/MENE0603248X.htm, consultée le 5 juillet 2017).

11. Il s'agissait notamment de Paris Diderot-Paris 7, Toulouse 2-Jean Jaurès, Lyon 2-Lumière, Lille 3-Charles-de-Gaulle, Reims Champagne-Ardenne, Montpellier 3-Paul Valéry, l'École des Arts et Métiers et ParisTech.

12. Par exemple, la convention interministérielle du 25 février 2000 (op. cit.) prenait soin de rappeler que « l'Union européenne (...) fait de l'égalité entre les femmes et les hommes l'un des axes prioritaires des politiques de l'emploi des États membres » et que «des fonds structurels européens seront d'ailleurs mobilisés pour financer les actions engagées en ce sens au niveau national et régional ».

13. Règlement (CE) n 1784/1999 du Parlement européen et du Conseil du 12 juillet 1999 relatif au Fonds social européen (JO L 213, 13.08.1999, p. 5).

14. Règlement (CEE) n ${ }^{\circ} 2084 / 93$ du Conseil du 20 juillet 1993 relatif au Fonds social européen (JO L 193, 31.07. 1993, p. 39). 
15. Sur ce point, v. Maria STRATIGAKI, «La politique du recul. De l'intégration de l'égalité 'des sexes' à l'intégration de l'égalité 'pour tous' ", Cahiers du genre, n 44, 2008/1, pp. 49-72.

16. Convention signée le 22 mars 2005, http://www.egalite-femmes-hommes.gouv.fr/wpcontent/uploads/2012/07/16_convention-cge.pdf, consultée le 5 juillet 2017.

17. Charte adoptée par l'assemblée plénière de la CPU le 17 décembre 2009, http://www.cpu.fr/ wp-content/uploads/2013/08/Charte_CPU_Egalite_Femme_Homme_17-12-2009.pdf.

18. Nous soulignons.

19. Isabelle KRAUS, Laure BONNAUD, Collette GUILLOPÉ, Laurence MARSALLE, «La Conférence Permanente des chargé.e.s de mission Égalité/Diversité (CPED) ou mission assimilée, des universités françaises ", in Le genre dans l'enseignement supérieur et la recherche. Livre Blanc de l'Association Nationale des Etudes Féministes, La Dispute, 2014, pp. 209-et ss.

20. V. Bertrand SEILLER, "Acte Administratif - Identification", Répertoire du contentieux administratif, Dalloz, oct. 2015, not. $n^{\circ} 266$ et ss.

21. Pour une délimitation de la notion, v. not. Michael Anca AILINCA, «Propos introductif ", in Soft law et droits fondamentaux. Actes du colloque du 4 et 5 février 2016, Pedone, 2017, pp. 7-27.

22. Les quatre autres engagements sont: «(...) 2. utiliser des outils de communication non sexistes, non discriminants, non stéréotypés ; 3 . (...) faire et diffuser un état des lieux statistique sexué ; 4. (...) organiser des actions de sensibilisation à l'égalité Femmes/Hommes auprès des étudiant-e-s comme du personnel ; 5. (...) prévenir toute forme de violence et de harcèlement ».

23. La Charte CPU/Cdefi prend soin d'ajouter que l'établissement s'engage à «lui donner les moyens de mettre en œuvre la politique définie ».

24. V. en introduction, supra.

25. Amendement $n^{\circ}$ AC 480 présenté par M. Sébastien Denaja, Mmes Catherine Coutelle, Ségolène Neuville et Maud Olivier au nom de la Délégation aux droits des femmes et à l'égalité des chances entre les hommes et les femmes de l'Assemblée nationale, Rapport de V. FELTESSE du 16 mai 2013, op.cit.

26. V. l'intervention de M. Patrick Hetzel, député Les Républicains, en commission des affaires culturelles et de l'éducation de l'Assemblée nationale, Rapport de V. FELTESSE du 16 mai 2013, op.cit.

27. Article $25 \mathrm{du}$ texte, http://www.assemblee-nationale.fr/14/ta-commission/r1042-a0.asp, consulté le 5 juillet 2017.

28. V. par ex. l'Université Paris-Diderot.

29. C'était pourtant une préoccupation de la ministre de l'enseignement supérieur, Geneviève Fioraso, qui avait rappelé lors de la discussion en plénière du projet de loi devant l'Assemblée nationale, la présence nécessaire d'une personne référente «à laquelle les étudiantes et les étudiants pourront s'adresser ", http://www.assemblee-nationale.fr/14/cri/2012-2013/20130249.asp, consultée le 5 juillet 2017.

30. En décembre 2011, la CPED répertoriait 36 missions égalité femmes-hommes (v. Isabelle KRAUS, et autres, «La Conférence Permanente des chargé.e.s de mission Égalité/Diversité (CPED) ou mission assimilée, des universités françaises ", op. cit.).

31. Chiffres obtenus à partir de l'annuaire de la CPED. Nous remercions Rozenn Texier-Picard, Présidente de la CPED, de nous avoir fourni ces données.

32. La Charte de 2013 peut également être un référentiel utile mais n'étant pas obligatoire, elle ne saurait engager l'intégralité des EPES.

33. Mesure 1 : coordination des politiques d'égalité hommes-femmes dans l'E.S.R.; Mesure 2 : mettre en œuvre la loi du 12 mars 2012 relative à l'accès à l'emploi titulaire et à l'amélioration des conditions d'emploi des agents contractuels dans la fonction publique, à la lutte contre les discriminations et portant diverses dispositions relatives à la fonction publique; Mesure 3 : mieux intégrer l'égalité professionnelle dans le déroulement de carrière; Mesure 4 : tendre vers 
la parité dans les instances représentatives; Mesure 5 : favoriser la mixité des formations et lutter contre les stéréotypes; Mesure $6:$ lutter contre les violences sexuelles; Mesure $7:$ soutenir et diffuser les recherches sur le genre ; Mesure $8:$ agir au niveau européen.

34. Plan d'action en ligne, http://www.enseignementsup-recherche.gouv.fr/cid70662/egaliteentre-les-femmes-et-les-hommes-plan-d-action-du-m.e.s.r.html, consultée le 5 juillet 2017.

35. Circulaire 2015-193 du ministère de l'éducation nationale, de l'enseignement supérieur et de la recherche parue au bulletin officiel de l'enseignement supérieur et de la recherche $n^{\circ} 44 \mathrm{du}$ 26-11-2015, NOR : MENS1522912C.

36. Selon l'article R. 712-33 du code de l'éducation «la commission d'instruction [de la section disciplinaire de l'établissement] instruit l'affaire par tous les moyens qu'elle juge propres à l'éclairer ». 37. Sur cette question, v. dans ce numéro spécial : Alexis ZARCA, « La répression disciplinaire du harcèlement sexuel à l'université », La Revue des droits de l'homme [En ligne], 12 | 2017, mis en ligne le 29 juin 2017, consulté le 12 juillet 2017. URL : http://revdh.revues.org/3109.

38. Circulaire du 25 novembre 2015 relative à la prévention et aux traitements du harcèlement sexuel dans les établissements publics d'enseignement supérieur et de recherche du MENESR.

39. Véronique CHAMPEIL-DESPLATS, «Le droit de la lutte contre les discriminations face aux cadres conceptuels de l'ordre juridique français », La Revue des droits de l'homme [En ligne], 9 | 2016, mis en ligne le 08 mars 2016, consulté le 05 juillet 2017. URL : http://revdh.revues.org/2049; DOI : 10.4000/revdh.2049.

40. V. par ex. Sandrine DAUPHIN et Réjane SÉNAC-SLAWINSKI (dir.), «Gender mainstreaming: de l'égalité des sexes à la diversité ? ", Cahier du genre, n 44, 2008/1, pp. 5-16.

41. V. sur ce point Le genre dans l'enseignement supérieur et la recherche, op. cit., pp. 17 et ss.

\section{RÉSUMÉS}

L'article L. 712-2 alinéa 10 du code de l'éducation, modifié par la loi Fioraso du 22 juillet 2013, dispose que le président de l'université «installe, sur proposition conjointe du conseil d'administration et du conseil académique, une mission 'égalité entre les hommes et les femmes'». Demeurée un temps à la discrétion des établissements publics d'enseignement supérieur et de recherche, la présence d'une «mission » égalité femmes-hommes est désormais obligatoire pour toutes les universités et un nombre croissant d'établissements d'enseignement supérieur. Cette institutionnalisation progressive de la cause des femmes dans le monde académique favorise-t-elle la lutte contre les inégalités entre les sexes ou s'agit-il d'une avancée en trompe l'œil ? La présente analyse des missions égalité femmes-hommes dans les universités se propose d'apporter des éléments d'explication susceptibles d'éclairer les raisons de la réussite ou, le cas échéant, de l'échec de ce dispositif en revenant, notamment, sur la genèse des missions égalité femmes-hommes à l'université, leurs fondements juridiques, l'évolution de leur nature et de leurs fonctions depuis les années 2000, avant qu'elles ne deviennent obligatoires, et en identifiant leurs forces et faiblesses.

The Education Code, amended by the Fioraso Law of 22 July 2013, stipulates that the president of the university " shall, on a joint proposal by the board of the University and the academic council, a mission 'equality between men and women' ". Remained a time at the discretion of public institutions of higher education and research, the "missions" equality between women and men is now compulsory for all universities and a growing number of higher education 
institutions. Does this gradual institutionalization of the cause of women in the academic world promote the struggle against gender inequalities or is this a step forward more apparent than real? The present analysis of the missions equality between women and men in universities proposes to examin the reasons for the success or, where appropriate, the failure of this mechanism by addressing the genesis of these missions at university, their legal foundations, the evolution of their nature and functions since the 2000s, before they became compulsory, and by identifying their strengths and weaknesses.

\section{INDEX}

Keywords : mission equality between women and men, Fioraso law, institutionalization, public institutions of higher education and research, european social fund, charter for equality between women and men in higher education and research institutions, conferences of the 'grandes écoles', conference of university presidents, conference of directors of french engineering schools, Permanent conference of mission head of equality and diversity, sexual harassment, gender mainstreaming, diversity mainstreaming

Mots-clés : mission égalité femmes-hommes, loi Fioraso, institutionnalisation, établissements publics d'enseignement supérieur et de recherche, Fonds social européen, charte pour l'égalité entre femmes et hommes dans les établissements d'enseignement supérieur et de recherche, conférences des grandes écoles, conférence des présidents d'université, conférence des directeurs des écoles françaises d'ingénieurs, conférence permanente des chargé(e)s de mission égalité et diversité, harcèlement sexuel, approche intégrée du genre, approche intégrée de la diversité

\section{AUTEUR}

\section{BÉATRICE DELZANGLES}

Béatrice Delzangles est Maitre de conférences à l'université Paris-Dauphine (CR2D) PSL

University Research, Référente égalité de l'université Paris Dauphine 